\title{
LA AUTONOMÍA DE LA PRODUCCIÓN DE CONOCIMIENTO COMO POLÍTICA DE LA UNIVERSIDAD*
}

\section{THE AUTONOMY OF KNOWLEDGE PRODUCTION AS UNIVERSITY POLICY}

\author{
JUAN MANUEL GARRIDO**, JUAN FELIPE ESPINOSA***, \\ TOMÁS UNDURRAGA ${ }^{* * * *}$
}

Resumen: Contra el punto de vista que caracteriza la universidad y el conocimiento que ella produce como emprendimientos generadores de utilidades, se propone defender la autonomía que ha caracterizado históricamente la investigación en la universidad. Tradicionalmente, la universidad ha sabido dejarse determinar por la legalidad que gobierna los procesos de producción de conocimiento, donde la utilidad social, política o económica no cuenta como operador determinante de sus resultados. Reconocer la especificidad de los procesos de producción de conocimiento es un requerimiento analítico básico para su gestión adecuada. La pregunta rectora para la gestión del conocimiento en la universidad no es cómo maximizar su utilidad, sino cómo asegurar la autonomía de los procesos que lo producen.

Palabras Clave: Autonomía, universidad, producción de conocimiento, capitalismo académico, política de investigación.

AвSTRACT: Contrary to the managerial perspective that characterizes the university and its production as a profit-generating enterprise, we propose to protect the exceptional and asymmetric autonomy that, historically, has characterized the university. Traditionally, universities have allowed to be determined by the legality that governs the processes of knowledge production. Where the social, political or economic usefulness does not count as an operator which determines its outcome. Recognizing the specificity of knowledge production is a fundamental analytical requirement for its proper management. The guiding question for research policies in universities is not

*Este artículo forma parte del proyecto Conicyt / PIA Anillo SOC180039, "Knowledge Production in Contemporary Chile", del proyecto Fondecyt Regular 1190543 "Doing Laboratory Studies in Chile: Re-engaging Science in the Making", y del proyecto Fondecyt de Iniciación 11180611 "Incendios forestales y nuevo régimen climático: interrogando la producción de conocimiento y valorización del medioambiente en expertos en bosques en el sur de Chile".

** Doctor en Filosofía. Departamento de Filosofía, Universidad Alberto Hurtado, Santiago, Chile. Correo electrónico: jgarrido@uahurtado.cl. Orcid: https://orcid.org/0000-0003-4509-1121.

*** Doctor en Management. Facultad de Economía y Negocios, Universidad Andrés Bello, Viña del Mar, Chile. Correo electrónico: juan.espinosa@unab.cl. Orcid: https://orcid.org/0000-0002-5629-6328.

**** Doctor en Sociología. Departamento de Sociología, Universidad Alberto Hurtado, Santiago, Chile. Correo electrónico: tundurraga@uahurtado.cl. Orcid: https://orcid.org/0000-0003-4267-5826. 
how to maximize the usefulness of knowledge, but how to ensure the autonomy of its production processes.

KeYwords: Autonomy, University, Knowledge Production, Academic Capitalism, Research Policy.

Recibido: 2019-07-15. Aceptado: 2020-03-04.

\section{LA HEGEMONÍA DE LA UNIVERSIDAD COMO INSTITUCIÓN PARA LA PRODUCCIÓN DE CONOCIMIENTO}

Tinguna institución rivaliza seriamente con la universidad en lo que 1 se refiere a producción de conocimiento. En la actualidad, proliferan organismos que buscan competir con ella, tales como centros de estudio, consultoras, think tanks, laboratorios de innovación, industrias especializadas, entre otros (Medvetz, 2014; Mirowski, 2013; Thrift, 2005). Estos organismos suelen hacer alarde de su eficiencia y mayor conexión con la esfera pública, de su capacidad de cultivar un conocimiento multidisciplinario y aplicado (Gibbons et al., 1994; Nowotny et al., 2003), en contraste con la supuesta inercia improductiva y autorreferente de la investigación universitaria tradicional, anclada en saberes codificados y en jurisdicciones disciplinares socialmente impertinentes y económicamente estériles.

Sin embargo, las formas tradicionales de producir conocimiento en la universidad no parecen sustituibles por la investigación que se produce en estos nuevos organismos. La universidad centraliza, estandariza y legitima las prácticas asociadas a la investigación, en todas las áreas. Las universidades siguen siendo las instituciones que generan la base científica y tecnológica para la innovación de ruptura (Giannopoulou et. al., 2019) y las que determinan el valor y el carácter de una industria -como se ha mostrado, por ejemplo, a propósito de la industria vitivinícola en Chile e Italia (Giuliani \& Arza, 2009).

Si se tiene en cuenta que ninguna universidad es capaz de generar de manera autónoma la totalidad de los recursos que necesita para financiar sus actividades, entonces lo razonable sería dudar de que la universidad pueda mantener su hegemonía en el ámbito de la investigación. Ninguna universidad puede prescindir de aportes estatales o privados. Muchas veces, se teme que estos aportes, con sus respectivas agendas y prioridades, condicionen de manera indebida las actividades de la universidad, incluida la investigación. El financiamiento externo instala temas de interés, pro- 
mueve estilos y formas de producir resultados, influye en contrataciones y carreras, pauta las destrezas laborales que deben generarse en los más jóvenes, estimula el desarrollo de algunas disciplinas en detrimento de otras, inhibe formas de transferencia científico-tecnológica, determina la estructura de los currículos, etcétera. Muchos consideran que la dependencia de financiamiento externo conduce a la destrucción de la universidad como institución para la producción de conocimiento (Granger, 2015). Los teóricos del nuevo institucionalismo, por ejemplo, sostienen que la investigación en las universidades ha degenerado en un emprendimiento que altera la valoración de la ciencia (Powell \& Colyvas, 2008; Powell \& DiMaggio, 1991). El conocimiento se mide en función del patentamiento y licenciamiento de los resultados, lo que habría llevado a un descuido generalizado de la investigación básica y del acceso público al conocimiento (Glenna et al., 2011; Radder, 2019).

Sin desconocer las dificultades provocadas por todos estos condicionamientos externos de la universidad, la posición que sostendremos en este ensayo busca ser más matizada y, por decirlo de alguna manera, menos pesimista, que la de autores como los recién citados. Sostendremos que los condicionamientos externos y amenazas a la autonomía universitaria, así como la emergencia de organismos competidores en investigación, pueden ciertamente afectar la creación y el funcionamiento de instituciones complejas con investigación de alto nivel, pero no desnaturalizan los procesos de producción de conocimiento. Si una universidad es "universidad" más que por su mero nombre, entonces ha debido crear las condiciones políticas, económicas y administrativas necesarias para que la producción de conocimiento tenga lugar. Nuestra opinión es que las intervenciones externas no impactan, en última instancia, en las reglas que gobiernan internamente los procesos de investigación como tales. Cualesquiera sean los intereses que la determinan, la investigación está sometida a reglas de índole específica, diferentes a las que gobiernan los ámbitos social, económico o político en que ella tendrá impacto.

En el presente ensayo propondremos lo siguiente. Si es verdad que la universidad concentra las actividades de producción de conocimiento y sienta los criterios que las definen internamente como tales, entonces la "universidad", lo que sea que ella sea y donde sea que ella tenga lugar, difícilmente responderá a las presiones del entorno social, político y económico del mismo modo en que lo hacen el resto de las instituciones de la sociedad. Esto tiene consecuencias para diseñar una política universitaria, y especialmente para diseñar una política de la investigación en la univer- 
sidad. La topología descentralizada de la universidad contemporánea ha conducido a promover su liberalización organizacional, una gestión que minimice planificación y verticalidad, similar a como ocurre en organizaciones comerciales e industriales. Por ejemplo, Jan Youtie \& Philip Shapira (2008) sugieren explotar las oportunidades que ofrecen nodos descentralizados que emergen en los puntos de interacción de la universidad con otras organizaciones. Se piensa que una universidad expuesta al mercado será más sensible a las necesidades epistémicas de la sociedad y más eficiente en la producción de conocimiento (Nowotny et al., 2001). Pero ello no siempre es el caso. En Chile, en particular, la mercantilización de las universidades no ha transformado ni mejorado las prácticas científicas, y en cambio sí se ha vuelto una amenaza para su fisionomía tradicional (Barandiaran, 2015). Por contra-intuitivo que parezca desde el punto de vista del management, a nosotros nos parece más razonable proteger la autonomía excepcional (Garrido et al., 2012) que ha caracterizado históricamente a la producción de conocimiento en la universidad, aun a costa de tener que tolerar su "verticalidad", "improductividad" e "ineficiencia" -si aceptamos hablar con los criterios evaluativos del managerialism.

Producir conocimiento es una actividad y misión irrenunciables de la universidad. Es de ese modo y no de otro como la universidad contribuye a la sociedad, y como se ha vuelto insustituible como institución. Es conocimiento lo que la sociedad necesita para producir la oportunidad de su propio desarrollo. Tal como lo mostraremos en la última sección de este trabajo (sección 4), la pregunta rectora para la gestión del conocimiento en la universidad no es cómo maximizar su utilidad, sino cómo asegurar la autonomía de los procesos que lo producen. Esto porque la utilidad del conocimiento para la vida no puede ser índice de éxito de la investigación universitaria. La utilidad -cuya percepción es esencial en la organización política y económica de toda sociedad- no es una propiedad definitoria del conocimiento ni un operador en el proceso de su producción (intentaremos mostrarlo en la sección 3 de este trabajo). Los beneficios del conocimiento en general son efectos no intencionados de la investigación. Conocimiento puede haberlo útil o inútil y es difícil, si no imposible, anticipar e incluso definir la utilidad o inutilidad de lo que existe como conocimiento. Estos enormes dinosaurios que llamamos universidades, incesantemente criticados y desacreditados, obsesivamente estudiados y vigilados, han sabido generar -aunque sea por inercia- una peculiar capacidad de dejarse determinar, y de manera esencial, por la legalidad que gobierna los procesos que producen conocimiento nuevo. 


\section{LA UNIVERSIDAD EN LA ÉPOCA DEL CAPITALISMO ACADÉMICO}

La universidad como institución que concentra las actividades de investigación tiene una relevancia especial en la sociedad contemporánea. Nuestras sociedades cifran nada menos que su propio desarrollo en el desarrollo de nuevo conocimiento. De acuerdo con estimaciones hechas por Youtie y Shapira (2008) acerca del sistema universitario norteamericano, entre 1954 y 1979 los gastos en I+D en la universidad crecieron a una tasa promedio de $8,1 \%$ anual, tasa significativamente más alta que la de 5,3\% anual para la economía norteamericana en su conjunto. En los 25 años siguientes (hasta 2004), la tasa de crecimiento de I+D en la universidad se redujo a $5.0 \%$ anual, pero siguió siendo superior a la de 3,9\% para el resto de la economía estadounidense. Seguramente no es fácil encontrar otros momentos históricos en que la universidad hubiera captado una cantidad semejante de recursos. La ampliación y democratización del sistema universitario también tuvo lugar en los Estados europeos. Después de la Segunda Guerra Mundial, en la mayoría de los países de Europa occidental, el Estado se convirtió en un importante contribuyente financiero al crecimiento de las instituciones de educación superior (Seville \& Tooley, 1997). Aunque en menor escala, los emergentes Estados de compromiso de los años cincuenta y sesenta en América Latina también ampliaron sus aportes a los sistemas universitarios, aunque en nuestra región serán las políticas de mercado de los ochenta en adelante las que realmente democratizarán el acceso a la universidad.

La producción de conocimiento es la actividad que define lo que entendemos por universidad. Ahora bien, debido a la importancia que dicha actividad tiene para el conjunto de la sociedad, lo razonable es esperar que los límites que definen la universidad se relativicen. Darle centralidad a la universidad como institución para la producción de conocimiento conduce a desdibujar sus fronteras con respecto a los demás instrumentos de acción económica, política y social. Como señala el filósofo francés Jacques Derrida (1994), "en una sociedad industrial e hiper-tecnificada, el espacio académico es menos que nunca el claustro monacal o monástico que por lo demás nunca fue realmente" (p. 24, trad. propia).

Es cierto que, históricamente, la universidad ha debido responder a las necesidades económicas de su entorno (por ejemplo, formando profesionales). Sin embargo, su peso y posición dentro de la sociedad ha cambiado a través del tiempo, así como las condiciones y grados de autonomía que goza para producir conocimiento. De acuerdo con la periodización propuesta 
por Brockliss (2000), en Europa hacia el 1400 había 40 universidades, 150 a principios del siglo XX y más de 500 en 1985. El número de estudiantes aumentó proporcionalmente en este período: si a principios del siglo XVII un 2,5\% de la población europea adulta (masculina) se formaba en la universidad, en el siglo XXI lo hace sobre el $30 \%$ de la población. Para el caso de América Latina, Rama (2006) muestra que la tasa de cobertura es del orden del 28,5\% en el año 2003, la que en 1950 alcanzaba solo un 2\% (López Segrera, 2008; Rama, 2006). América Latina pasó de tener 75 universidades en 1950 a 3.000 en el 2010, con una matrícula multiplicada por setenta veces en dicho período (Fernandez Lamarra, 2015). Debe notarse que la ampliación de recursos y la democratización del acceso de la universidad es concomitante con la importancia creciente que ha tenido el conocimiento en nuestras sociedades.

Ahora bien, desde el punto de vista de los grados de autonomía universitaria, el modelo de universidad inspirada en Humboldt es, tal vez, el que ofrece las condiciones de investigación y docencia más avanzados. De acuerdo con Baert \& Shipman (2005), el ideal de universidad propuesto por el humanista decimonónico inspiró el desarrollo de las instituciones de educación superior durante los siglos XIX y XX:

En su forma ideal-tipo, la noción humboldtiana describe a la universidad como un cuerpo independiente de profesionales autónomos, responsables y supervisados por sí mismos. Profesores y académicos son supuestamente autónomos de los intereses de clase u otros tipos, y hablan y escriben con autoridad y posición crítica... Existe una clara frontera entre la academia y el mundo exterior, esta última sujeta a las fuerzas del mercado y la primera inmunizada contra estos mecanismos... Las actividades intelectuales de los académicos supuestamente tienen valor en sí mismas ya que están dirigidos hacia los ideales centrales de la sociedad, como la autoedificación (Bildung). Estos esfuerzos académicos podrían tener beneficios prácticos -spin-offs- para la sociedad, pero eso es precisamente lo que son, efectos no intencionados. No hay expectativa de que el profesor humboldtiano cree conocimiento con un valor práctico inmediato y visible. (Baert \& Shipman, 2005, p. 159, trad. propia)

Este modelo universitario inspiró la expansión de las instituciones de educación superior contemporáneas, y tuvo su apogeo en la segunda mitad del siglo XX, especialmente en los países del norte con la consolidación del financiamiento estatal. Si bien muchos académicos conciben este concepto de universidad como una visión utópica, imposible de mantener frente a las 
presiones actuales por producir conocimiento útil y contable, conectado a necesidades de la industria y de la política pública, las universidades que se asemejan más a esta noción humboldtiana se encuentran entre las más prestigiosas del mundo.

El modelo humboldtiano no ha estado exento de críticas. Por un lado, se ha observado que promueve una visión elitista de la educación superior, al alcance de solo una pequeña fracción de la sociedad. El modelo humboltiano reproduciría arbitrariamente las distinciones culturales entre alta y baja cultura (Bourdieu, 1998). Por otro lado, este modelo de universidad ha sido cuestionado desde el managerialism o 'new public management', como un tipo de organización poco eficiente, poco transparente, poco "accountable", que mejoraría si respondiera a las exigencias de mercado que rigen en las instituciones privadas.

Esta última crítica, en particular, ha puesto creciente presión a la universidad para integrar criterios de eficiencia y productividad en su funcionamiento, responder a necesidades profesionales de la industria, y democratizar su acceso a nuevos públicos. El acceso a fondos de financiamiento de investigación y el desarrollo de la carrera de los propios académicos están crecientemente condicionados a su capacidad de adaptarse a las demandas externas. Según Baert \& Shipman (2005, p. 168), estas presiones "de mercado" tendrían a la universidad 'sitiada'. En particular, la cultura de auditoría (Power, 1997; Strathern, 2000), en lugar de brindar eficiencia en la producción de conocimiento al interior de la universidad, sería especialmente nociva al introducir criterios de competencia individual entre académicos, erosionar la confianza colegial entre pares, y afectar la independencia académica con criterios externos al objetivo de investigación. Paradójicamente, entonces, la implementación de sistemas para mayor eficiencia contravino la base original del "management", que consiste en la búsqueda de medios eficaces para alcanzar objetivos definidos. De acuerdo con Baert \& Shipman (2005), las "externalidades" de la cultura de auditoría y la exposición de la academia a las presiones del mercado tienen consecuencias nefastas para la producción de conocimiento:

En primer lugar, conduce a una doble mercantilización del conocimiento. Los proyectos de investigación no solo tendrán más éxito con los organismos de financiamiento si de alguna manera prometen un valor en efectivo al final del camino; además, estos proyectos se convierten en una mercancía al interior de la unidad académica misma. Hacen que los académicos involucrados se salgan de la enseñanza, empleen asistentes de investigación, etc. En segundo lugar, los investigadores principales 
se ven obligados a adoptar modelos de gestión para ejecutar grandes proyectos, para supervisar personas que trabajan para ellos y para controlar las finanzas involucradas. El éxito en el mundo académico se ha vuelto cada vez más dependiente de las cualidades que tradicionalmente se asociaban con las empresas: administrar proyectos, dirigir personas, motivar equipos de trabajo. En tercer lugar, hay claras ramificaciones a nivel de identidad profesional. Mientras que en la idea humboldtiana los académicos tenían una noción relativamente estable de identidad, centrada en una vocación hacia la disciplina y la tradición, los organismos que hoy distribuyen los fondos tratan de redirigir constantemente la investigación hacia las demandas siempre cambiantes de la sociedad. (p. 175, trad. propia)

Slaughter \& Leslie (2001) han llamado a las presiones de mercado un "capitalismo académico" (p. 154). Con este término, se refieren a comportamientos de mercado por parte de las universidades y académicos, como la competencia por recursos provenientes de fuentes externas o las actividades encaminadas a generar ingresos con fines de lucro, el patentamiento, el royalty y las licencias, la creación de compañías derivadas y la generación de asociaciones universidad-industria para vender servicios educacionales. La nuestra es una universidad post- o híper-industrial diseminada en el tejido económico de la sociedad, un hub de conocimiento involucrado intensamente en el desarrollo regional, la innovación, y el comercio (Youtie \& Shapira, 2008).

\section{PRODUCCIÓN E INSTITUCIONALIZACIÓN DEL CONOCIMIENTO}

La defensa y justificación sociales del conocimiento se basan en la idea de que este puede y debe usarse para el bienestar del ser humano. Se asume que el ser humano tiene en sus propias manos la tarea y la capacidad de agenciarse las condiciones para la propia subsistencia y desarrollo. El objetivo de toda política científica consiste en generar las condiciones para que el conocimiento pueda convertirse en una prestación útil. Se busca "organizar la ciencia como una empresa para la invención e institucionalizarla socialmente para que las invenciones científicas sean puestas al servicio del ser humano" (Böhme, 1993, p. 10, trad. propia).

Uno de los presupuestos básicos de la institucionalidad científica moderna es que los logros de la ciencia y la tecnología son, en lo esencial, logros humanos que pueden ser puestos a disposición del ser humano para fines propiamente humanos. El "dominio" sobre la naturaleza es tal, 
un dominio, porque el ser humano se muestra capaz de hacer prevalecer su propia existencia como fin por sobre el poderío ciego de la causalidad natural, convertida en medio gracias a los avances de la ciencia y la tecnología. Ahora bien, no es seguro que el ser humano haya demostrado ser realmente capaz de darle al conocimiento una estructura intencional. No se trata solo de constatar las dificultades peculiares que enfrenta el diseño de buenas políticas públicas en materia de ciencia y tecnología. Se trata de algo bastante más serio: ¿acaso no vivimos en una época en que la "causalidad natural" es todo menos funcional a los intereses humanos? Lo que hoy llena de incertidumbre y ensombrece la fabricación tecnocientífica de la vida es justamente efecto del uso que hacemos de los logros de la ciencia y la tecnología. El antropoceno o capitaloceno (Moore, 2016), es decir, la época geológica marcada por el significativo impacto humano en los ecosistemas de la Tierra (Klein, 2014; Latour, 2017), evidencia los límites físicos del planeta frente a la explotación creciente de sus recursos por los avances del conocimiento tecnocientífico. Ningún sistema causal, por mucho que sea visto y usado por el ser humano en vista del ser humano, puede generar otra regla que la de su propia reproducción; pero esta reproducción no incluye una finalidad, los objetos que produce no tienen la estructura de un fin, lo que quiere decir que pueden terminar reproducidos en cualquier contexto y sirviendo a cualquier fin.

Además, una mutación sustantiva parece haber ocurrido en nuestra relación con la ciencia y la tecnología. Hemos dejado de usar el conocimiento solo para mejorar la vida y hemos pasado a tener que usarlo para posibilitarla. La vida humana, en todas sus formas y niveles, simplemente ya no es posible sin generación de conocimiento científico y tecnológico. Es decir, ya no es posible suponer que la vida humana es algo dado y que, sobre la base de eso dado, el conocimiento es capaz de introducir mejoras. Hoy la producción de conocimiento es la única posibilidad que tiene el ser humano de no dejar de existir. Todo el sentido de la vida parece subsumido en la tarea de generar las condiciones para no dejar de ser. La ciencia y la tecnología se han transformado en un instrumento para sobrevivir (Böhme, 1993, p. 24).

Como observa Böhme (1993), se supone que la ciencia y la tecnología nos aliviarían del trabajo de generar las condiciones materiales de la vida; sin embargo, han causado todo lo contrario: hoy nos vemos obligados a generar todo lo que antes "recibíamos" de la naturaleza (abrigo, alimento, salud, etc.). La producción de la vida por medio de la ciencia y la tecnología nos ha conducido a un punto donde toda nuestra capacidad productiva 
debe orientarse a producir las condiciones para no dejar de producir. Como dice Gérard Granel (1973):

nadie se preocupa ya sino de producir la posibilidad misma de vivir, posibilidad que cada uno de nosotros persigue por medio de su forma de vestirse, de su relación con el dinero, su práctica del deseo o de su denegación, sus estudios o su relación con el mundo, su militancia política o su desilusión. (trad. propia)

Vivimos hoy una encrucijada apremiante. El problema de gestionar el conocimiento nunca había sido tan relevante políticamente. En el conocimiento ciframos la posibilidad misma de no dejar de ser. Sin embargo, ni la vida ni cualquier otro fin en general tienen la capacidad de condicionar la producción de conocimiento. Todo conocimiento útil para la vida es una consecuencia no buscada por la producción de conocimiento. En efecto, de todas las formas de producción humana, el conocimiento es la única en que el fin se desconoce al momento de diseñar e implementar el proceso de su producción. No hay manera de anticipar el sistema de elementos y actividades que engendrarán un conocimiento nuevo.

Por una parte, cualquier conocimiento en cualquier área puede ser usado para cualquier fin, es decir, ningún conocimiento puede evitar ser instrumentalizado, para los mejores o peores fines. Por otra parte, ningún resultado se obtiene completamente de un proceso diseñado en función de dicho resultado pensado como fin. El fin y, en general, los intereses -institucionales, gremiales o individuales-inmiscuidos en los procesos de investigación, no son realmente operativos. Su eficacia causal es limitada o nula.

Sin duda, la investigación no sería lo que es sin los componentes teleológico-agenciales que la intervención humana incorpora. No podemos abstraer a los investigadores de su pertenencia a una cultura, a una corporalidad, a las reglas institucionales y un mundo vividos que determinan consciente o inconscientemente lo que ellos son capaces de percibir, imaginar, simbolizar, representar, etc. Pero tampoco podemos confundir estas estructuras con las reglas de funcionamiento de los sistemas (materiales o simbólicos, experimentales o teóricos) que permiten visibilizar y manipular objetos. El investigador está siempre obligado a reducir la arbitrariedad de su intervención a la luz de las estructuras y lógicas que gobiernan el desarrollo de dichos sistemas. Debe entrenarse para entender qué puede y no qué quiere conocer. Qué puede y no qué quiere preguntar. Debe aprender cómo su imaginación, su saber, su experiencia y su intuición pueden volverse funcionales a los sistemas que producen conocimiento. 
Hay una suerte de materialismo inherente a la práctica científica. Una ideología puede ser funcional o disfuncional a la instalación social y al progreso de la ciencia. Por ejemplo, la idea instalada en Europa durante el siglo XIX de que debemos y podemos transformar el entorno natural condujo al desarrollo de conocimientos de las materias, como la química y las ingenierías. Alternativamente, en Chile contemporáneo parecen seleccionarse y estimularse los conocimientos que mejoren las tecnologías de extracción, más que transformación, de las materias. Pero la productividad científica, la capacidad que tiene la ciencia de producir sistemas de objetos controlables, reproducibles, manipulables, no requiere de la legitimación de relatos aceptados colectivamente (o por las elites y grupos de poder en una sociedad). La ciencia remite a prácticas y procesos sui generis que no obedecen a las lógicas con que se generan los campos sociales en que ella actúa. Como lo observa el historiador de la ciencia Hans-Jörg Rheinberger (2018):

Hemos entrado en una época en que la ciencia, sobre todo con su base tecnológica, ha desarrollado una dinámica propia, de modo que ya no necesita de la superestructura ideológica. La ciencia es hoy, y en virtud de su propia existencia material, un factor social sui generis. En tanto que investigación, y solo para dar un ejemplo, garantiza y expande nuestro indispensable universo de medicamentos. La ciencia se ha convertido en algo extremadamente esencial a nuestra realidad social y, por lo tanto, su funcionamiento ya no depende de si al Papa le gustan o no las declaraciones de Darwin. (p. 107, trad. propia).

Todo ello tiene consecuencias significativas desde el punto de vista de una política de la universidad como institución para la producción de conocimiento. Las instituciones del conocimiento albergan actividades que, en el fondo, son profundamente indiferentes a los fines con que estas mismas instituciones interpretan el sentido de la vida social. Pero eso es exactamente lo que las define como instituciones de conocimiento: tienen como fin exponerse a la ausencia de fin, confiarse a lo desconocido. Aun si acordamos que existe una misión para las instituciones del conocimiento y que esta misión consiste en producir medios de producción, su vocación de instituciones del conocimiento las conduce a quedar expuestas a la generación de lo desconocido, es decir, la generación de lo que puede ser disfuncional a la producción de los medios de producción, de lo que puede producir pérdida, inercia, improductividad y ocio (como ocurre, frecuentemente, en la universidad).

Cuando se pasa por alto la estructura no intencional de los procesos de 
producción de conocimiento, se corre el riesgo de inhibirlos, de suprimirlos, y junto con ello de suprimir la razón de ser misma de su institucionalización. Igualmente, cuando se pasa por alto la necesidad de institucionalizar de forma adecuada la producción de conocimiento, también ponemos en riesgo la posibilidad de producirlo. Las estructuras no intencionales de la investigación no tienen oportunidad de subsistir fuera de una institucionalidad que las cobije y proteja. El desafío para una política del conocimiento consiste en articular estos elementos opuestos y contradictorios.

\section{LA AUTONOMÍA UNIVERSITARIA COMO POLÍTICA PARA LA PRODUCCIÓN DE CONOCIMIENTO}

Es imprescindible priorizar las actividades de investigación en función de lo que percibimos como temas sociales y económicos urgentes, objetivos estratégicos, ventajas comparativas, etc. Sin embargo, ninguna priorización, por pertinente que sea, generará la base científica (humana y no humana, simbólica, tecnológica o material) que permita realizar una determinada política. Para priorizar, para utilizar el conocimiento o sacarle el provecho que queremos, primero hay que contar con la capacidad de generarlo. Y esa capacidad se genera instalando sistemas cuyo funcionamiento es totalmente contingente respecto de lo que busque cualquier política científica. Por ejemplo, la propuesta de "clusters dinámicos e innovadores" del exministro de Hacienda Nicolás Eyzaguirre (Eyzaguirre et al., 2005, p. 51) suena muy razonable, y eficiente, pero condenada al fracaso si primero no se asegura la generación y renovación permanentes de una base científica sólida, diversa y creativa.

Lo razonable para un país como el nuestro es diseñar una política orgánica, atenta a la complejidad y al dinamismo de las formas de producir conocimiento, potenciando de manera equilibrada programas e instrumentos de distinto carácter (investigación individual y asociativa, disciplinar y multidisciplinar, básica y aplicada, por curiosidad y por misión, formación de capital humano y carrera de investigador, etc.). Tal como lo muestra la actual literatura sobre planificación estratégica (van der Heijden, 2005), no se trata de crear el futuro, sino de enfrentarlo, de crear las condiciones adecuadas para propiciar el encuentro de actores y la generación de sistemas de objetos e instrumentos con los que se pueda hacer frente a lo que no conocemos. En cambio, pensar que el conocimiento se puede "adoptar y adaptar" (Eyzaguirre et al., 2005, pp. 21, 49 y 51) según los desafíos del presente constituye un error. Error que por lo demás impacta ante todo a 
las economías emergentes que no cuentan con una robusta base científica local (Qiu et al., 2017).

Ya sea por diseño, ya sea por inercia, por historia o trayectoria, la universidad es la única institución para la producción de conocimiento que parece definida por la tarea de inventar formas de organización que hagan convivir las estructuras intencionales y las no intencionales que componen el ámbito de la investigación. Por un lado, nuestra caracterización del conocimiento como proceso autónomo respecto de los fines de la vida social conduce a pensar que no puede haber incompatibilidades de principio entre la producción de conocimiento y la mercantilización del mundo académico. Por otro lado, sin embargo, la mera compatibilidad entre los fines exógenos que aportan la industria y el comercio, y los requerimientos endógenos para la producción de conocimiento no es garantía de una institucionalidad sana y productiva. Como vimos al inicio de este ensayo, se ha reportado ampliamente que la intromisión excesiva del entorno económico, político y social merma la producción y transferencia pública del conocimiento. Es cierto que la cultura de la auditoría y de la eficiencia, la mercantilización del conocimiento y la orientación a su utilidad y aplicación, ayudan a que la universidad goce de mayor legitimación política, económica y social. Sin embargo, estas presiones externas también distorsionan el entorno académico y perjudican la producción de conocimiento. Y al dañar los procesos de producción de conocimiento, se daña a la larga la propia industria y el comercio. Como lo advierte Martin Kenney (1988):

quizás la mayor ironía será experimentada por la propia industria (...). En la medida en que la universidad sea comprada y parcelada, la ciencia básica va a sufrir crecientemente. El académico especulativo y no comercial estará en desventaja, y los bienes intelectuales imprescindibles para producir una fuerza laboral capacitada, así como el lugar de nacimiento de nuevas ideas, serán erosionados y contaminados. La industria descubrirá entonces que, al ser incapaz de controlarse a sí misma, acostumbrada a no tener restricciones de parte del sector público, habrá contaminado su propio lugar de cultivo. (p. 246, trad. propia)

Absorber en la utilidad la legalidad de los procesos que producen conocimiento, o viceversa, terminaría destruyendo tanto el conocimiento como su utilidad. No es viable ni deseable purificar la investigación de las exigencias y la evaluación del entorno, pues probablemente la investigación terminaría perdiendo la protección política, legal e institucional que necesita para existir. Pero si la sociedad no toma nota de los límites que 
la naturaleza del quehacer académico le inflige, y en general del hecho de que no es la agencia social la que produce el conocimiento que se necesita producir, entonces corre el riesgo de terminar aniquilando aquello mismo que ella debe organizar y de donde proviene la oportunidad para su propio desarrollo como sociedad. Una institucionalidad científica, por poderosa que sea, no tiene poder alguno sobre los microprocesos que engendran conocimiento. La legalidad indómita que gobierna estos procesos es quizás una porción pequeña, acaso mínima, de lo que llamamos "conocimiento", pero es insustituible y de ella depende que se produzca lo que la sociedad necesita que se produzca.

Reconocer la existencia de estas legalidades diferentes o contradictorias es un requerimiento analítico básico para la gestión adecuada del conocimiento en la universidad y, por extensión, en la sociedad. La convivencia de ambas necesita ser regulada para ser virtuosa. Afortunadamente, esa regulación no necesita imaginarse desde cero: la autonomía que las actividades tradicionales y propiamente académicas de la investigación alojadas en las universidades reclaman permanentemente para sí puede ser considerada como el principal marcador de dicha regulación.

Hasta cierto punto, la universidad es el lugar en que la sociedad toma conciencia de los límites infranqueables que tiene para producir y gestionar la posibilidad de sí misma y de su porvenir como sociedad. Actividades tan abstractas y aparentemente improductivas como la matemática pura o la filología generan una experiencia bastante concreta de que "resolver problemas" es una actividad obligada a la paciencia y al estudio. Lo que menos importa es la solución, que será efecto no intencionado del estudio. Importa entrenarse a reconocer y a producir lo que no está, lo que está por venir o por inventar. En la universidad medieval, por ejemplo, el estudio de los textos canónicos no tenía el propósito de reproducir lo conocido; se trataba más bien de un mecanismo para despertar la interpretación creativa y el pensamiento crítico (piénsese en la "quaestio disputata") (Fisch, 2015; Rexroth, 2018). La universidad es la experiencia concreta de que la cultura misma se forma de esta manera: lidiando con lo desconocido.

La universidad es una institución organizada casi por inercia en función de la producción de conocimiento: desde el acto incondicional del estudio hasta la investigación y la formación de investigadores y de profesionales. Muchas veces se quiere combatir la inercia, la aparente falta de ductilidad de la universidad, su poca agilidad para adaptarse al mercado, su apego irracional a las disciplinas, a los currículos y a los ritos tradicionales. Pero es eso lo que hace que la universidad sea una institución única para la pro- 
ducción de conocimiento (todo tipo de conocimientos) y sobre todo para la producción de los criterios mismos con los que en general la sociedad reconoce un conocimiento y a quienes participan de su producción.

Desde sus inicios, la universidad y los universitarios (estudiantes y académicos) reclaman para sí altos grados de autonomía política, jurídica y económica, respecto de la Iglesia tanto como del Estado, aun cuando al mismo tiempo comprometen servicios fundamentales al resto de la sociedad (Fisch, 2015). Pero conviene hacer una distinción entre la autonomía de los universitarios, la así llamada libertad académica, y la autonomía de los procesos que producen conocimiento. La regulación de la universidad no pasa solamente por garantizar la autorregulación de las comunidades de universitarios. Estas se organizan igual que cualquier otro grupo social, a saber, de acuerdo con fines, intenciones o intereses. No por el hecho de dedicarse al estudio los animales académicos tienen menos intereses que la empresa o la Iglesia. Incluso muchas veces la autonomía otorgada a los universitarios puede ir en detrimento de la autonomía requerida por los procesos de producción de conocimiento. Los investigadores no son inmunes a ideologías o filosofías espontáneas acerca de sus propias prácticas (Althusser, 1974), a la luz de las cuales seleccionan y validan lo que vale como conocimiento, y también a quienes lo producen.

Ahora bien, por importante que sea distinguir entre la autonomía de los investigadores y la autonomía de la investigación, es razonable pensar que disminuir la primera impacta negativamente en la segunda, por cuanto limita la adaptabilidad de los investigadores al funcionamiento de los sistemas, procesos y microprocesos que producen conocimiento. La libertad académica encuentra en ello una justificación importante. En la actualidad, un elemento delicado que fragiliza la libertad académica proviene del hecho de que los académicos son trabajadores asalariados, hipersensibles a la competitividad interna y a los incentivos externos. Es poco o nada lo que el régimen de propiedad de las universidades, o incluso la misión e idearios que las definen, repercutiría en la libertad académica si existiera, por ejemplo, un estatuto laboral excepcional de los investigadores en determinadas fases de su carrera. La protección legal extraordinaria ha sido un rasgo distintivo de las comunidades académicas desde la fundación de las universidades (Fisch, 2015). En sistemas como el norteamericano, donde la universidad tiene amplia interacción con el mercado, existen instrumentos como la "permanencia" (tenure), que contribuyen a resguardar niveles significativos de libertad académica. A este respecto, el sistema universitario chileno parece empeñado en romper con la tradición. 


\section{REFERENCIAS}

Althusser, L. (1974). Philosophie et philosophie spontanée des savants, 1967. Paris: F. Maspero.

Baert, P. \& Shipman, A. (2005). University under siege? Trust and accountability in the contemporary academy. European Societies, 7(1), 157-185.

Barandiaran, J. (2015). Chile's Environmental Assessments: Contested Knowledge in an Emerging Democracy. Science as Culture, 24(3), 251-275.

Böhme, G. (1993). Am Ende des Baconschen Zeitalters: Studien zur Wissenschaftsentwicklung. Franfurt am Main: Suhrkamp Verlag.

Bourdieu, P. (1998). The State Nobility: Elite Schools in the Field of Power. Cambridge: Stanford University Press.

Brockliss, L. (2000). Gown and Town: The University and the City in Europe, 1200-2000. Minerva, 38(2), 147-170.

Derrida, J. (1994). Force de loi: Le "Fondement mystique de l'autorité." Paris: Galilée.

Eyzaguirre, N., Marcel, M., Rodriguez, J., y Tokman, M. (2005). Hacia la economía del crecimiento: El camino para crecer con equidad en el largo plazo. Estudios Públicos, 97, 5-57.

Fernandez Lamarra, N. (2015). El desarrollo de la educación superior en América Latina en perspectiva comparada. En M. C. Corrochano, A. Marrero, \& A. Otero (eds.), Educación superior en perspectiva comparada y regional (pp. 21-32). Buenos Aires: Teseo.

Fisch, S. (2015). Geschichte der europäischen Universität: Von Bologna nach Bologna. München: C.H.Beck.

Garrido, J. M., Herrera, H. y Svensson, M. (2012). La excepción universitaria. Reflexiones sobre la educación superior en Chile. Santiago: Ediciones UDP.

Giannopoulou, E., Barlatier, P-J., \& Pénin, J. (2019). Same but different? Research and Technology Organizations, Universities and the Innovation Activities of Firms. Research Policy, 48(1), 223-233.

Gibbons, M., Limoges, C., Nowotny, H., Schwartzman, S., Scott, P., \& Trow, M. (1994). The New Production of Knowledge: The Dynamics of Science and Research in Contemporary Societies. London: Sage.

Giuliani, E. \& Arza, V. (2009). What drives the Formation of 'valuable' University-Industry Linkages?: Insights from the Wine Industry. Research Policy, 38(6), 906-921.

Glenna, L., Welsh, R., Ervin, D., Lacy, W., \& Biscotti, D. (2011). Commercial science, scientists' values, and university biotechnology research agendas. Research Policy 40(7), 957-968. https://doi.org/https://doi.org/10.1016/j.respol.2011.05.002. http://www.sciencedirect.com/science/article/pii/S00487 33311000679.

Granger, C. (2015). La destruction de l'université française. Paris: La Fabrique.

Granel, G. (1973). Cours sur Gramsci, Boukharine et Bordiga. 1973-1974. Retrieved from http://www.gerardgranel.com/txt_pdf/3-Cours_Gramsci.pdf 
Kenney, M. (1988). Biotechnology: The University Industrial Complex. New Haven: Yale University Press.

Klein, N. (2014). This Changes Everything: Capitalism vs. the Climate. New York: Simon \& Schuster.

Latour, B. (2017). Facing Gaia: Eight Lectures on the New Climatic Regime (1 edition; C. Porter, Trans.). Cambridge/Medford: Polity.

López Segrera, F. (2008). Tendencias de la educación superior en el mundo y en América Latina y el Caribe. Avaliação: Revista da Avaliação da Educação Superior, 13(2), 267-291.

Medvetz, T. (2014). Think Tanks in America. Chicago: University of Chicago Press.

Mirowski, P. (2013). Never Let a Serious Crisis Go to Waste: How Neoliberalism survived the financial Meltdown. London/New York: Verso.

Moore, J. W. (ed.). (2016). Anthropocene or Capitalocene?: Nature, History, and the Crisis of Capitalism. Oakland: PM Press.

Nowotny, H., Scott, P. \& Gibbons, M. (2001). Re-Thinking Science: Knowledge and the Public in an Age of Uncertainty. London: Polity Press.

Nowotny, H., Scott, P., \& Gibbons, M. (2003). Introduction: 'Mode 2' revisited: The new Production of Knowledge. Minerva, 41(3), 179-194.

Powell, W. W. \& Colyvas, J. A. (2008). Microfoundations of Institutional Theory. In The SAGE Handbook of Organizational Institutionalism (pp. 276-298). Londres/California: Sage Publications.

Powell, W. W. \& DiMaggio, P. J. (1991). The New Institutionalism in Organizational Analysis. Chicago: University of Chicago Press.

Power, M. (1997). The Audit Society: Rituals of Verification. London: Oxford University Press.

Qiu, S., Liu, X. \& Gao, T. (2017). Do emerging countries prefer local knowledge or distant knowledge? Spillover Effect of University collaborations on local Firms. Research Policy, 46(7), 1299-1311. https://doi.org/10.1016/j.respol.2017.06.001

Radder, H. (2019). From the Commodification to the Common Good: Reconstructing Science, Society and Technology. Pittsburg: University of Pittsburg Press.

Rama, C. (2006). La tercera reforma de la educación superior en América Latina y el Caribe: Masificación, regulaciones e internacionalización. Revista Educación y Pedagogía, 18(46), 13-24.

Rexroth, F. (2018). Fröhliche Scholastik: Die Wissenschaftsrevolution des Mittelalters (2nd ed.). München: C.H. Beck.

Rheinberger, H.-J. (2018). Experimentalität: Hans-Jörg Rheinberger im Gespräch über Labor, Atelier und Archiv (1st ed.). Berlin: Kulturverlag Kadmos.

Seville, A., \& Tooley, J. (1997). The Debate on Higher Education. London: Institute of Economic Affairs and Education and Training Unit.

Slaughter, S., \& Leslie, L. L. (2001). Expanding and Elaborating the Concept of Academic Capitalism. Organization, 8(2), 154-161. 
Strathern, M. (ed.). (2000). Audit Cultures: Anthropological Studies in Accountability, Ethics and the Academy. London/New York: Routledge.

Thrift, N. (2005). Knowing Capitalism. Londres: Sage Publications.

van der Heijden, K. (2005). Scenarios: The Art of Strategic Conversation. Londres: Wiley.

Youtie, J., \& Shapira, P. (2008). Building an innovation hub: A Case Study of the Transformation of University Roles in regional technological and economic Development. Research Policy, 37(8), 1188-1204. 